\title{
ON CYCLIC NUMBERS OF ONE-DIMENSIONAL COMPACT SETS*
}

\author{
BY \\ W. A. WILSON
}

1. An interesting class of one-dimensional compact sets is that of cyclically connected sets. P. Alexandroff $\dagger$ has defined the cyclic numbers of such sets and obtained important properties. It is the main purpose of this article to obtain a theorem on the divisor of a sequence and a generalization of Alexandroff's addition theorem. The proofs are based on a modification of Alexandroff's definition of cyclic number, which seems to the writer to furnish a more natural approach for those whose main interest is the theory of point sets. The work is confined to compact metric spaces.

2. A finite set of closed sets $\left\{d_{i}\right\}$, of which no three have common points and none is a sub-set of the union of the others, is called a canonical set of cells. If the set $M$ can be expressed as the union of a canonical set of cells, each of which has a diameter less than some positive $\sigma$, we say that these cells constitute a canonical $\sigma$-covering of $M$. If $M$ is compact and one-dimensional at most, it is well known that there is a canonical $\sigma$-covering of $M$ for each positive $\sigma$. If $M$ is contained in the canonical set $S=\left\{d_{i}\right\}$ and $M \cdot d_{i} \neq 0$ for each $i$, we call $S$ a canonical enclosure.

If the cell $d_{i}$ has points in common with $w_{i}$ other cells, $w_{i}$ is called the order of $d_{i}$. A component of the covering is a maximal sub-set of the cells which cannot be separated into two sets such that no cell of one set contains points of the other set.

If $p$ is the number of components,

$$
m=\frac{1}{2} \sum_{1}^{n}\left(w_{i}-2\right)+p=\frac{1}{2} \sum_{1}^{n} w_{i}-n+p
$$

is called the cyclic number of the covering or enclosure. $\ddagger$ The number $I$ defined by

$$
I=\frac{1}{2} \sum_{1}^{n}\left(w_{i}-2\right)=m-p
$$

* Presented to the Society, September 9, 1931; received by the editors July 11, 1931.

$\dagger \ddot{U} b e r$ kombinatorische Eigenschaften allgemeiner Kurven, Mathematische Annalen, vol. 96, pp. 512-554.

$\ddagger$ It is apparent from $\$ 11$ of the article referred to above that $m$ is the same as the $s(L)$ of the corresponding, linear complex used by Alexandroff. 
is called the index of the covering or enclosure. The numbers $m$ and $I$ will be used sometimes when the covering is not canonical. In this connection it should be noted that, if the covering satisfies the definition of being canonical, except for the existence of one or more cells each of which is a sub-set of some other cell, the canonical covering resulting from the deletion of these cells has the same cyclic number as the original one. Such a covering may be called almost canonical.

In reckoning $m, p$, and $I$, void cells are not counted. Thus, if no two cells have common points, $I=-n=-p$ and $m=0$. At times we use $w\left(d_{i}\right), m(S)$, $p(S)$, or $I(S)$ for definiteness.

3. Theorem. Let $M$ be a compact set imbedded in a compact space $Z$ and $\left\{d_{i}\right\}$ be a canonical $\sigma$-covering or -enclosure. Then there is an $\epsilon>0$ such that, if $e_{i}$ is the set of points of $Z$ whose distances from $d_{i}$ are not greater than $\epsilon$, then $e_{i} \cdot e_{j} \neq 0$ if and only if $d_{i} \cdot d_{j} \neq 0$, and the set of cells $\left\{e_{i}\right\}$ is a canonical $\sigma$-enclosure of $M$ of the same cyclic and component numbers as $\left\{d_{i}\right\}$.

There is a positive $\delta$ less than one-third the least distance between any pair of disjoint cells $\left\{d_{i}\right\}$. Since the given set is canonical and the number of cells is finite, there is a positive $\eta$ such that every $d_{i}$ contains a point whose distance from the union of the remaining cells is greater than $\eta$. The conclusion is then valid if $\epsilon$ is less than both $\delta$ and $\eta$, and $2 \epsilon$ is less than $\sigma$ minus the upper bound of the diameters of the cells $\left\{d_{i}\right\}$. For on account of the first statement $e_{i} \cdot e_{j} \neq 0$ if and only if $d_{i} \cdot d_{j} \neq 0$. On account of the second statement no $e_{i}$ is contained in the union of the others. Finally the enclosure is a $\sigma$-enclosure because the diameter of $e_{i}$ exceeds that of $d_{i}$ by at most $2 \epsilon$.

4. Theorem. Let $A$ and $B$ be canonical sets of cells such that the point sets which they form are disjoint. Then $m(A+B):=m(A)+m(B)$ and $I(A+B)$ $=I(A)+I(B)$.

COROLlaRy. The cyclic number or index of a canonical set of cells is the sum of the cyclic numbers or indices, respectively, of the components.

5. Theorem. Let $A=\left\{d_{i}\right\}$ be a canonical set of cells. To each $d_{i}$ let there correspond a closed sub-set $e_{i}$ and let $B$ be a canonical set of the cells $\left\{e_{i}\right\}$. Then $m(B) \leqq m(A)$.

First let $A$ be connected and its cells arranged in such an order that the first $n$ form a connected set $A_{n}$. Let $B_{n}$ be the sub-set of $B$ corresponding to cells of $A_{n}$ and assume that $m\left(A_{n}\right) \geqq m\left(B_{n}\right)$.

If $e_{n+1}$ is void, it is obvious that $m\left(A_{n+1}\right) \geqq m\left(B_{n+1}\right)$. If $e_{n+1}$ is not void, let it meet $c$ components of $B_{n}$ in a total of $k$ cells. It is easily seen that 
$I\left(B_{n+1}\right)=I\left(B_{n}\right)+k-1$ and $p\left(B_{n+1}\right)=p\left(B_{n}\right)-c+1 ;$ hence $m\left(B_{n+1}\right)=m\left(B_{n}\right)$ $+k-c$. Now $d_{n+1}$ may meet more than $k$ cells of $A_{n}$; hence $m\left(A_{n+1}\right) \geqq m\left(A_{n}\right)$ $+k-1$. Clearly $k-1 \geqq k-c$, unless $c=0$. In this case $k=0$, but on the other hand $d_{n+1}$ meets at least one cell of $A_{n}$. Thus in every case the addition of $e_{n+1}$ and $d_{n+1}$ increases $m\left(A_{n}\right)$ by at least as much as $m\left(B_{n}\right)$ is increased. Hence the theorem is true by induction.

The case that $A$ is not connected follows at once by $\S 4$.

Corollary 1. Let $A=\left\{d_{i}\right\}$ be a canonical set of cells and $B \subset A$. Then $m(B) \leqq m(A)$.

For it is obvious that $B$ is canonical and this corollary is the special case of the theorem where $e_{i}=d_{i}$ or $e_{i}$ is void.

COROLLARY 2. If in addition to the hypotheses of the above theorem or corollary we know that $p(B) \geqq p(A)$, then $I(B) \leqq I(A)$.

COROLLARY 3. Let $M$ be a compact set and $\left\{d_{i}\right\}$ be a canonical enclosure of $M$ of cyclic number m. Let $e_{i}=M \cdot d_{i}$. Then there is a sub-set of the cells $\left\{e_{i}\right\}$ which is a canonical covering of $M$ of cyclic number not greater than $m$.

For the deletion of any cell $e_{i}$ which is a sub-set of the union of the other cells leaves a covering of $M$. If this is done successively, we finally reach a sub-set of the cells $\left\{e_{i}\right\}$ which is a canonical covering of $M$ and the theorem is then applicable.

6. If $A=\left\{d_{i}\right\}$ and $B=\left\{e_{k}\right\}$ are two canonical sets of cells which have common points and $A+B$ is canonical, a discussion of $A+B$ requires the assignment of a meaning to $A \cdot B$. By $A \cdot B$ we shall mean the set of cells $\left\{f_{r}\right\}$ which are either common cells of $A$ and $B$ or divisors of a cell of $A$ not in $B$ and a cell of $B$ not in $A$. If $f_{r}$ is a cell of the first type and $f_{s}=d_{i} \cdot e_{k}$ is one of the second, $f_{r} \cdot f_{s}=0$, because $A+B$ is canonical. In like manner no two cells of the second type can have common points. Thus $A \cdot B$ is canonical and each component is either a cell of the second type or a connected set of cells of the first type. It is also a simple matter to show by induction that, with the above definitions,

$$
p(A+B)+p(A \cdot B)-p(A)-p(B) \geqq 0 .
$$

7. Theorem. Let $A, B$, and $A+B$ be canonical collections of cells. Then

$$
I(A+B)=I(A)+I(B)-I(A \cdot B) .
$$

Let $B_{0}$ be the set of cells common to $A$ and $B$; let there be $n$ other cells of $B$ and let $B_{k}$ be the union of $B_{0}$ and the first $k$ of these cells $\left\{e_{i}\right\}$. Suppose that the theorem is true for $A+B_{k}$; i.e., 


$$
I\left(A+B_{k}\right)=I(A)+I\left(B_{k}\right)-I\left(A \cdot B_{k}\right) .
$$

This is obviously true when $k=0$, since $A \cdot B_{0}=B_{0}$.

Let $e_{k+1}$ meet $c$ cells of $B_{0}, a$ cells of $A-B_{0}$, and $b$ cells of $B_{k}-B_{0}$. As the sets are canonical, this gives at once

$$
\begin{aligned}
I\left(A+B_{k+1}\right) & =I\left(A+B_{k}\right)+a+b+c-1 ; \\
I\left(B_{k+1}\right) & =I\left(B_{k}\right)+b+c-1 .
\end{aligned}
$$

By $\$ 6$ we see that the addition of $e_{k+1}$ adds $a$ isolated cells to $A \cdot B_{k}$; hence

$$
I\left(A \cdot B_{k+1}\right)=I\left(A \cdot B_{k}\right)-a .
$$

Relations (2), (3), and (4) make (1) true for $k+1$. Thus the theorem is true by induction.

Corollary 1. If $A+B$ is a canonical collection of cells,

$$
m(A+B)=m(A)+m(B)-m(A \cdot B)+p(A+B)-p(A)-p(B)+p(A \cdot B) .
$$

Corollary 2. If $A+B$ is a canonical collection of cells,

$$
m(A+B) \geqq m(A)+m(B)-m(A \cdot B) .
$$

Remark. The above theorem and the one in $\$ 14$ are variants of the index formulas derived by S. Straszewicz (Über die Zerschneidung der Ebene durch abgeschlossene Mengen, Fundamenta Mathematicae, vol. 7, pp. 159-187) for plane sets. The index used here is not, however, the same. This theorem is easily generalized to cover the case where the set of cells is not canonical and may indeed be taken as a basis to define the cyclic number.

8. A compact metric set $M$ is called at least $m$ cyclic if for a positive $\sigma$ small enough every canonical $\sigma$-covering of $M$ has its cyclic number at least $m$. It is called at most $m$ cyclic if for every positive $\sigma$ there is some canonical $\sigma$-covering of cyclic number not more than $m$. If $M$ is imbedded in a compact space, we can of course substitute the word enclosure for covering without altering the sense of these definitions.

We say that $M$ is $m$ cyclic if it is both at most and at least $m$ cyclic; i.e., if for some $\sigma>0$ every canonical $\sigma$-covering has its cyclic number at least $m$ and for every $\sigma>0$ some canonical $\sigma$-covering has the cyclic number $m$. If for every integer $m, M$ is at least $m$ cyclic, we say that it is $\infty$ cyclic. It is a simple matter to show from these definitions that every (at most) one-dimensional compact set has a definite cyclic number, finite or infinite. It is also clear from page 523 of the reference given in $\$ 1$ that $m$ is one less than Alexandroff's $\kappa$.

9. It is readily seen with the aid of $\S 5$, Corollary 3 , that, if $M$ and $N$ are one-dimensional compact sets and $M \subset N$, the cyclic number of $M$ is not more 
than that of $N$. (Cf. Alexandroff, loc. cit., p. 523.) Hence the divisor of a decreasing sequence of at most $m$ cyclic one-dimensional compact sets is at most $m$ cyclic, and every set of this character contains a set irreducible with respect to these properties. But the first of these statements is seen to become false and the second doubtful, if the word most is replaced by least, and so the question of the cyclic number of the divisor of a sequence requires further investigation.

Similarly, the relation between the cyclic number of a set and those of its components is obvious when the component number is finite, but requires a proof when the component number is infinite. We begin with the second question.

10. THEOREM. Let $M$ be a compact one-dimensional set. If its cyclic number is finite, it is the sum of the cyclic numbers of those components having positive cyclic numbers.

We know by $\$ 7$ that for every partition of $M$ into $r$ disjoint closed sets $\left\{M_{i}\right\}, m(M)=\sum_{1} m\left(M_{i}\right)$. There is then a largest $r \leqq m$ such that for each $i$, $m\left(M_{i}\right) \geqq 1$.

Now no such $M_{i}$ can contain more than one component $K_{i}$ of positive cyclic number. If $M_{i}$ is decomposed into disjoint closed sets $P_{i}$ and $Q_{i}$, where $K_{i} \subseteq P_{i}$, it is clear that $m\left(P_{i}\right)=m\left(M_{i}\right)$ and $m\left(Q_{i}\right)=0$. If this can be done for each $i$ so that $K_{i}=P_{i}$, the theorem is evident'y proved.

In the other event there is a decreasing sequence $\left\{P_{i j}\right\}$ of closed sub-sets of $M_{i}$ having the component $K_{i}$ as its divisor, such that $m\left(P_{i j}\right)=m\left(M_{i}\right)$ for each $j, Q_{i j}=M_{i}-P_{i j}$ is closed, and $m\left(Q_{i j}\right)=0$. Let $\sigma$ be so small that every canonical $\sigma$-covering of $M_{i}$ has its cyclic number at least $m\left(M_{i}\right)$. Let $\delta<\sigma / 2$ and let $A=\left\{d_{n}\right\}$ be a canonical $\delta$-covering of $K_{i}$ of cyclic number $m\left(K_{i}\right)$. By $\$ 3$ we can take $\epsilon<\sigma / 6$ and so small that, if $e_{n}$ is the set of points of $M_{i}$ whose distances from $d_{n}$ are not greater than $\epsilon$, the set of cells $\left\{e_{n}\right\}$ is a canonical $\sigma$-enclosure of some $P_{i j}$ of cyclic number $m\left(K_{i}\right)$, since $K_{i}$ is the divisor of $\left\{P_{i j}\right\}$. Setting $f_{n}=e_{n} \cdot P_{i j}$, there is by $\$ 5$, Corollary 3 , a sub-set $B$ of cells $\left\{f_{n}\right\}$ which is a canonical $\sigma$-covering of $P_{i j}$ of cyclic number not greater than $m\left(K_{i}\right)$. Let $C=\left\{g_{k}\right\}$ be a canonical $\sigma$-covering of $Q_{i j}$ of cyclic number 0 . Then $B+C$ is a canonical $\sigma$-covering of $M_{i}$ of cyclic number not more than $m\left(K_{i}\right)$. Hence' in this case also $m\left(K_{i}\right)=m\left(M_{i}\right)$, and the theorem is proved.

11. Theorem. Let $M$ be an $\infty$ cyclic compact one-dimensional set. Then either some component of $M$ is $\infty$ cyclic or an infinity of components have positive cyclic numbers.

We first show that the assumption that all the components are 0 cyclic 
leads to a contradiction. There is a dyadic decomposition* $M=\sum M_{i_{1}}=\sum M_{i_{1} i_{2}}$ $=\sum M_{i_{1} i_{2} i_{3}}$, etc., into disjoint closed sets, such that each component $K$ of $M$ is the divisor of some monotone descending sequence $\left\{M_{n}\right\}$, where $M_{n}$ is some $M_{i_{1} i_{2}} \ldots i_{n}$.

Take $\sigma>0$. There is a canonical $(\sigma / 2)$ - covering of $K, K=U\left[d_{r}\right]$, whose cyclic number is 0 . By $\S 3$ we can take $\delta<\sigma / 6$ and so small that, if $e_{r}$ is the set of points of $M$ whose distances from $d_{r}$ are not greater than $\delta$, the set of cells $\left\{e_{r}\right\}$ is a canonical $\sigma$-enclosure of $K$ of cyclic number 0 . For some $n_{k}$, this enclosure contains every $M_{n}, n \geqq n_{k}$, of the sequence whose divisor is $K$. That is, for each $K$ there is an $n_{k}$, such that there is a canonical $\sigma$-covering of cyclic number 0 of every $M_{n}, n \geqq n_{k}$, of the sequence whose divisor is $K$.

We now prove that $n_{k}$ has an upper bound for all components $\{K\}$. If this is not true, it follows by well known theorems that there is a sequence $\left\{K_{u}\right\}$ of components such that $n_{k_{u}} \rightarrow \infty$ and a $K$ containing the upper closed limiting set of $\left\{K_{u}\right\}$, as $u \rightarrow \infty$. But for $u$ large enough, $K_{u}$ and $K$ lie in the same $M_{n k}$; whence $n_{k_{u}} \leqq n_{k}$.

Let then $n$ be the upper bound of $n_{k}$. There is then a canonical $\sigma$-covering of each $M_{n}$ of cyclic number 0 . As these are disjoint and their number is finite, we have a canonical $\sigma$-covering of $M$ of cyclic number 0 . Since $\sigma$ was arbitrary, we see that $M$ is 0 cyclic, contrary to hypothesis. Hence not all the components of $M$ are 0 cyclic.

Now let $M$ have $s$ components $\left\{K_{i}\right\}$, each of cyclic number $m_{i}$, and let every other component be 0 cyclic. Taking any $\sigma>0$, let $K=U\left[d_{1, k}\right]$ be a canonical $(\sigma / 2)$-covering of $K_{1}$ of cyclic number $m_{1}$. Choose a positive $\delta$ so small that, if $e_{1, k}$ is the set of points of $M$ whose distances from $d_{1, k}$ are not more than $\delta$, then the set of cells $\left\{e_{1, k}\right\}$ is a canonical $\sigma$-enclosure of $K_{1}$ of cyclic number $m_{1}$. We can set $M=P_{1}+Q_{1}$, where $P_{1}$ and $Q_{1}$ are disjoint closed sets, $K_{1} \subseteq P_{1}, \sum_{2}^{s} K_{i} \subseteq Q_{1}$, and $P_{1}$ is contained in the enclosure $\left\{e_{1, k}\right\}$. Hence by $\$ 5$, Corollary 3 , this enclosure generates a canonical $\sigma$-covering of $P_{1}$ of cyclic number at most $m_{1}$.

In the same way $Q_{1}$ is the sum of disjoint closed sets $P_{2}$ and $Q_{2}$, such that $K_{2} \subseteq P_{2}, \sum_{3}^{s} K_{i} \subseteq Q_{2}$, and there is a canonical $\sigma$-covering of $P_{2}$ of cyclic number at most $m_{2}$. Finally we reach $Q_{s}=M-\sum_{1}^{8} P_{i}$. This has no component of positive cyclic number and so there is a canonical $\sigma$-covering of $Q_{s}$ of cyclic number 0 . Thus there is for any $\sigma$ a canonical $\sigma$-covering of $M$ of cyclic number at most $m^{\prime}=\sum_{1}^{s} m_{i}$. This is contrary to the hypothesis that $M$ is $\infty$ cyclic, and so the theorem is proved.

Remark. We can combine the results of these two sections in the state-

* F. Hausdorff, Mengenlehre, p. 131. 
ment that the cyclic number of any compact one-dimensional set is the sum of the cyclic numbers of its components, if we understand that the sum of any number of zeros is zero and that of any number of infinities is infinity. The cardinal number of the cyclic components may be that of any closed set.

12. LeMMA. Let $M$ and $M^{\prime}$ be compact one-dimensional sets lying in a compact space $Z, M^{\prime} \subseteq M$, and $M^{\prime}$ have $p^{\prime}$ components, $p^{\prime}$ finite. Let every canonical $\sigma$-enclosure of $M$ have its cyclic number at least $m$ and for some $\delta<\sigma / 2$ let there be a canonical $\delta$-covering of $M^{\prime}$ of cyclic number $m^{\prime}$ and component number $p^{\prime}$. Then there is an $\epsilon>0$ and an $\eta>0$ such that, if $E=\left\{e_{k}\right\}$ is any canonical $\eta$ enclosure of $M$ and $B$ is a sub-set of $E$ for which $M^{\prime} \subset B \subset V_{\epsilon}\left(M^{\prime}\right)^{*}$, while the other cells of $E$ form a set $C$ containing no point of $M^{\prime}$, then

$$
m(E)-m(B)+p(B) \geqq m-m^{\prime}+p^{\prime} .
$$

Let $D=\left\{d_{i}\right\}$ be the canonical $\delta$-covering referred to above. Let $\epsilon$ be so small that, if the set of points of $Z$ whose distances from $d_{i}$ are not greater than $2 \epsilon$ is taken as a cell, the set of such cells forms a canonical $\sigma$-enclosure of $M^{\prime}$ of cyclic number $m^{\prime}$ and component number $p^{\prime}$. (See $\$ 3$.) Let $\epsilon<\sigma / 6$ and $\eta<\epsilon / 2$. Let $E=\left\{e_{k}\right\}$ be any canonical $\eta$-enclosure of $M$ and $B$ be a subset of $E$ for which $M^{\prime} \subset B \subset V_{\epsilon}\left(M^{\prime}\right)$ and the other cells form a set $C$ such that $M^{\prime} \cdot C=0$. Then each cell $e_{k}$ of $B$ contains a point of $V_{\epsilon}\left(d_{i}\right)$ for some $i$.

Let $\Gamma_{i}$ be the union of the cells of $B$ containing points of $V_{\epsilon}\left(d_{i}\right)$. Then $\Gamma_{i} \cdot \Gamma_{j} \neq 0$ if and only if $d_{i} \cdot d_{j} \neq 0$, and $\left\{\Gamma_{i}\right\}$ is a canonical $\sigma$-enclosure of $M^{\prime}$ of cyclic number $m^{\prime}$ and component number $p^{\prime}$.

For each $i$ let $\Delta_{i}$ be the union of the cells $\left\{e_{k}\right\}$ contained in $\Gamma_{i}$, but in no $\Gamma_{j}, j>i$. The set $A$ of non-void cells $\left\{\Delta_{i}\right\}$ is a canonical enclosure of $M^{\prime}$ since $\Delta_{i} \subseteq \Gamma_{i}, m(A) \leqq m^{\prime}$ by $\S 5$, and $p(A) \geqq p^{\prime}$. It is easy to see that $A+C$ is a canonical $\sigma$-ençlosure of $M$, and we must prove the inequality given in the statement of the theorem.

We know that

(1) $m(A+C)-m(A)+p(A)=m(C)-m(A \cdot C)+p(A+C)-p(C)+p(A \cdot C)$;

(2) $m(B+C)-m(B)+p(B)=m(C)-m(B \cdot C)+p(B+C)-p(C)+p(B \cdot C)$.

Since $C$ has no cells in common with $A$ or $B, m(A \cdot C)=m(B \cdot C)=0$. As $A+C$ is obtained by combining certain cells of $B+C$ into new cells, $p(B+C) \geqq p(A+C)$. Each component of $A \cdot C$ is the divisor of a cell $e_{k}$ of $C$ and a cell $\Delta_{i}$ of $A$ and is therefore the sum of the divisors of $e_{k}$ and one or more cells $\left\{e_{i}\right\}$ contained in $\Delta_{i}$. As no two cells $\left\{\Delta_{i}\right\}$ contain common cells $\left\{e_{i}\right\}$, it follows that $p(B \cdot C) \geqq p(A \cdot C)$.

* This notation signifies the set of points of $Z$ whose distances from $M^{\prime}$ are less than $\epsilon$. 
Thus the left member of (2) is greater than or equal to that of (1). Since $m(A+C) \geqq m$ and $p(A) \geqq p^{\prime}$, while $m(A) \leqq m^{\prime}$, this proves the theorem.

CoROLlary 1. Under the hypotheses of the above theorem there is an $\eta>0$ such that, if $E=\left\{e_{k}\right\}$ is any canonical $\eta$-enclosure of $M$ and $B$ is the sub-set of cells containing points of $M^{\prime}$, then $m(E)-m(B) \geqq m-m^{\prime}$.

For in this case $p(B)=p^{\prime}$.

Corollary 2. Let $M$ and $M^{\prime}$ be compact one-dimensional sets with cyclic numbers $m$ and $m^{\prime}$, let $p^{\prime}=p\left(M^{\prime}\right)$ be finite, and let $M^{\prime} \subset M$. Then there is an $\epsilon>0$ and an $\eta>0$, such that, if $E=\left\{e_{k}\right\}$ is any canonical $\eta$-enclosure of $M$ and $B$ is a sub-set of $E$ for which $M^{\prime} \subset B \subset V_{\epsilon}\left(M^{\prime}\right)$, while the other cells of $E$ form a set $C$ containing no point of $M^{\prime}$, then $m(E)-m(B)+p(B) \geqq m-m^{\prime}+p^{\prime}$.

For it is easy to show that the hypotheses of the theorem apply here.

COROLlary 3. Under the hypotheses of Corollary 2 there is an $\eta>0$ such that, if $E=\left\{e_{k}\right\}$ is any canonical $\eta$-enclosure of $M$ and $B$ is the sub-set of cells containing points of $M^{\prime}$, then $m(E)-m(B) \geqq m-m^{\prime}$.

For again $p(B)=p^{\prime}$.

13. TheOREM. Let $\left\{M_{i}\right\}$ be a decreasing sequence of compact one-dimensional sets, each of finite cyclic number $m$, and let $M$ be the divisor of the sequence. Then $m$ is the cyclic number of $M$.

By $\$ 10$ each $M_{i}$ contains a sub-set $N_{i}$ consisting of the components of $M_{i}$ whose cyclic numbers are positive and $m\left(N_{i}\right)=m$. As there can be at most $m$ components in each $N_{i}$ and, if $A \subset B, m(A) \leqq m(B)$, we can assume without loss of generality that each $N_{i}$ consists of $p$ components $\left\{K_{i j}\right\}$, each of cyclic number $m_{j} \geqq 1$, where $p \leqq m$ and $m=\sum_{1}^{p} m_{j}$. Moreover, the sequence $\left\{N_{i}\right\}$ is monotone decreasing. Let $N$ be its divisor.

It is easily seen from $\$ 8$ that for each $i$ there is a greatest $\sigma_{i}$ such that every canonical $\sigma_{i}$-covering of $N_{i}$ has its cyclic number at least $m$. If the lower bound $\sigma$ of $\sigma_{i}$ is positive, we proceed as follows. Let $m^{\prime}=m(N)$ and $\left\{d_{n}\right\}$ be a canonical covering of $N$ of cyclic number $m^{\prime}$ and norm $\sigma / 2$. Taking $\epsilon$ small enough, we know that, if the set of points of $N_{1}$ whose distances from the cell $d_{n}$ are not greater than $\epsilon$ is taken as a cell, then the set of such cells forms a canonical $\sigma$-enclosure of $N$ and $N_{i}$, for $i$ large enough, of cyclic number $m^{\prime}$. This enclosure generates a canonical $\sigma$-covering of $N_{i}$ of cyclic number at most $m^{\prime}$, by $\S 5$. This is a contradiction unless $m^{\prime}=m$.

Let $\rho$ be less than the distance between any two components of $N_{1}$, and therefore of any $N_{i}$. If the lower bound of $\sigma_{i}$ is 0 , we may assume that every 
$\sigma_{i}<\rho / 3$ and that every $\sigma_{i}>3 \sigma_{i+1}$, since this is true for a partial sequence. If $\delta$ is slightly less than $\sigma_{i} / 2$, it is greater than $\sigma_{i+1}$. By definition of $\sigma_{i+1}$ there is a canonical $\delta$-covering of $N_{i+1}$ of cyclic number not greater than $m-1$ and of component number $p$. Then by $\$ 12$ there is an $\eta_{i}$ such that every canonical $\eta_{i}$-covering of $N_{i}$ has its cyclic number at least 1 larger than that of the subset of cells containing points of $N_{i+1}$. Let $r$ be any integer and $\eta$ be less than any $\eta_{i}, i \leqq r$.

Let $S_{1}$ be an $\eta$-covering of $N_{1}$ and $T_{2}$ be the set of cells of $S_{1}$ containing points of $N_{2}$. By $\S 5$, Corollary 3 , there is a set $S_{2}$ of cells which are divisors of $N_{2}$ and cells of $T_{2}$, and which forms a canonical $\eta$-covering of $N_{2}$ of cyclic number not greater than $m\left(T_{2}\right)$. Then by $\$ 12$,

$$
m\left(S_{1}\right) \geqq m\left(T_{2}\right)+1 \geqq m\left(S_{2}\right)+1 .
$$

Defining $T_{3}$ and $S_{3}$ in like manner, we have

$$
m\left(S_{2}\right) \geqq m\left(S_{3}\right)+1 .
$$

Finally we reach

$$
m\left(S_{r}\right) \geqq m\left(S_{r+1}\right)+1 .
$$

Relations (1), (2), $\cdots,(r)$ give

$$
m\left(S_{1}\right) \geqq r .
$$

Hence for every integer $r, m\left(N_{1}\right) \geqq r$, which is impossible by the hypothesis. Therefore the lower bound of $\sigma_{i}$ cannot be zero and $m(N)=m$. Since $N \subseteq M$ and $M \subseteq M_{i}$ for every $i, m(M)=m$, which was to be proved.

Corollary. Let $M$ be a one-dimensional compact set of finite cyclic number $m$. Then $N$ contains a set $K$ irreducible with respect to the properties of being closed and of cyclic number $m$.

14. TheOREM. Let $A$ and $B$ be one-dimensional compact sets, each having $a$ finite number of components and a finite cyclic number. Let $A \cdot B$ have a finite number of components. Then

(1) $m(A+B)=m(A)+m(B)-m(A \cdot B)+p(A+B)-p(A)-p(B)+p(A \cdot B)$.

There is a $\sigma$ so small that every canonical $\sigma$-enclosure of $A, B, A \cdot B$, or $A+B$ has the same component number and at least as great a cyclic number as the respective set. Let $D=\left\{d_{i}\right\}$ be a canonical $(\sigma / 2)$-covering of $A \cdot B$ of cyclic number $m(A \cdot B)$. Let $\epsilon<\sigma / 6$ and so small that $\$ 3$ is valid for the covering $D$ and the set $A \cdot B$ considered as imbedded in the space $A+B$. Let $\eta<\epsilon$ and so small that $\S 12$, Corollary 1 , is valid for $A$ and $A \cdot B$, and for $B$ and 
$A \cdot B$. Let $E=\left\{e_{k}\right\}$ be a canonical $\eta$-covering of $A$ of cyclic number $m(A)$; let $E^{\prime \prime}$ denote the set of cells of $E$ which contain points of $A \cdot B$, and $E^{\prime}$ the others. Similarly define $F=\left\{f_{k}\right\}, F^{\prime \prime}$, and $F^{\prime}$ with respect to $B$ and $A \cdot B$. Then $E^{\prime \prime}$ and $F^{\prime \prime}$ have the same cyclic and component numbers as $A \cdot B$.

Let $\Delta_{i}^{\prime}$ be the union of the cells $\left\{e_{k}\right\}$ containing points of $d_{i}$ but of no $d_{j}$, $j>i$. Let $\Delta_{i}^{\prime \prime}$ be the union of the cells $\left\{f_{k}\right\}$ containing points of $d_{i}$ but of no $d_{j}$, $j>i$, and $\Delta_{i}=\Delta_{i}^{\prime}+\Delta_{i}^{\prime \prime}$. Then $G=\left\{\Delta_{i}\right\}$ is a canonical $\sigma$-enclosure of $A \cdot B$, $m(G)=m(A \cdot B)$, and $p(G)=p(A \cdot B)$, by the choice of $\epsilon, \eta$, and $\sigma$.

Now $G+E^{\prime}$ is a canonical $\sigma$-enclosure of $A, G$ and $E^{\prime}$ have no common cells, $p\left(G+E^{\prime}\right)=p(A)$, and $m\left(G+E^{\prime}\right) \geqq m(A)$. Compare the equations

$$
\begin{aligned}
m\left(G+E^{\prime}\right) & =m(G)+m\left(E^{\prime}\right)+p\left(G+E^{\prime}\right)-p(G)-p\left(E^{\prime}\right)+p\left(G \cdot E^{\prime}\right) ; \\
m(E) & =m\left(E^{\prime \prime}\right)+m\left(E^{\prime}\right)+p\left(E^{\prime \prime}+E^{\prime}\right)-p\left(E^{\prime \prime}\right)-p\left(E^{\prime}\right)+p\left(E^{\prime \prime} \cdot E^{\prime}\right) .
\end{aligned}
$$

It is seen as in the proof of $\$ 12$ that $p\left(G \cdot E^{\prime}\right) \leqq p\left(E^{\prime \prime} \cdot E^{\prime}\right)$. As the other pairs of corresponding terms on the right are equal, this gives $m\left(G+E^{\prime}\right) \leqq m(E)$; whence $m\left(G+E^{\prime}\right)=m(A)$. Likewise, $G+F^{\prime}$ is a canonical $\sigma$-enclosure of $B$, $m\left(G+F^{\prime}\right)=m(B)$, and $p\left(G+F^{\prime}\right)=p(B)$.

Consider the canonical $\sigma$-covering $E^{\prime}+G+F^{\prime}$ of $A+B$. Since $E^{\prime} \cdot F^{\prime}=0$, we have at once from the above results and $\S 7$, Corollary 1 ,

$m\left(E^{\prime}+G+F^{\prime}\right)=m(A)+m(B)-m(A \cdot B)+p(A+B)-p(A)-p(B)+p(A \cdot B)$.

This shows that $m(A+B)$ is not greater than the right side of (1).

Now for any covering $D$ of $A+B$ and any sub-set $C$ we let $C_{D}$ be the set of cells containing points of $C$. We first note that $A_{D} \cdot B_{D}$ consists solely of cells common to $A_{D}$ and $B_{D}$. For, if a cell $d$ of $A_{D}$ meets a cell $d^{\prime}$ of $B_{D}$ and $d$ contains no point of $B, d \subset A$, and $d^{\prime}$ contains a point of $A$. Then $d^{\prime} \subset A_{D}$ and so $d \cdot d^{\prime}$ is deleted by our original definition of the divisor of two canonical collections of cells.

For any $\epsilon>0$ there is a $\delta>0$ so that for any canonical $\delta$-covering $A \cdot B \subset A_{D} \cdot B_{D} \subset V_{\epsilon}(A \cdot B)$ and no other cell of $(A+B)_{D}$ contains points of $A \cdot B$. As $A \cdot B$ is a sub-set of $A$, there is an $\epsilon>0$ and an $\eta>0$ for which $\$ 12$, Corollary 2 , is valid.

Also, for some $\sigma>0$ and less than both $\delta$ and $\eta$, every canonical $\sigma$-covering of $A+B$ is such that $p(A+B)_{D}=p(A+B), p\left(A_{D}\right)=p(A), p\left(B_{D}\right)=p(B)$, $m\left(A_{D}\right) \geqq m(A)$, and $m\left(B_{D}\right) \geqq m(B)$, and for some such covering $m(A+B)_{D}$ $=m(A+B)$. By $\S 7$,

$$
\begin{aligned}
m(A+B)_{D}=m\left(A_{D}\right)+m\left(B_{D}\right) & -m\left(A_{D} \cdot B_{D}\right) \\
& +p(A+B)_{D}-p\left(A_{D}\right)-p\left(B_{D}\right)+p\left(A_{D} \cdot B_{D}\right) .
\end{aligned}
$$


By $\$ 12$, Corollary $2, \quad m\left(A_{D}\right)-m\left(A_{D} \cdot B_{D}\right)+p\left(A_{D} \cdot B_{D}\right) \geqq m(A)-m(A \cdot B)$ $+p(A \cdot B)$. Hence the above equation shows that $m(A+B)$ is greater than or equal to the right hand side of (1). As we have already shown that it is not greater, the theorem is proved.

15. If we define the index of a set $A$ by $I(A)=m(A)-p(A)$, the above addition formula becomes $I(A+B)=I(A)+I(B)-I(A \cdot B)$, which is the same as the formula of Straszewicz and is readily generalized. For completeness the following rather obvious result is added.

Theorem. Let $A$ and $B$ be compact one-dimensional sets, let each have a finite set of components, and let $A \cdot B$ have an infinite set of components. Then $m(A+B)$ is infinite.

For some component $K$ of $A$ and some component $L$ of $B, K \cdot L$ has an infinite set of components. Then $K \cdot L$ is the sum of $r$ disjoint closed sets for any integer $r$. Hence for some $\sigma>0$ every canonical $\sigma$-covering $M+N$ of $K+L$, where $M$ denotes the set of cells containing points of $K$ and $N$ those containing points of $L$, is such that $M \cdot N$ has at least $r$ components. By $\S 7$,

$$
\begin{aligned}
m(M+N)=m(M)+m(N)- & m(M \cdot N) \\
& +p(M+N)-p(M)-p(N)+p(M \cdot N) .
\end{aligned}
$$

Obviously, $m(M) \geqq 0, m(N)-m(M \cdot N) \geqq 0$, and $p(M+N)=p(M)=p(N)=1$. Hence $m(M+N) \geqq r-1$. As $r$ was any integer, $m(K+L)$ is infinite; and so, a fortiori, $m(A+B)$ is infinite.

YALE UNIVERSITY, New Haven, Conn 